TITLE:

Existence of Selfsimilar Shrinking Curves for Anisotropic Curvature Flow Equations(Variational Problems and Related Topics)

$\operatorname{AUTHOR}(S)$ :

Mizoguchi, Noriko

CITATION:

Mizoguchi, Noriko. Existence of Selfsimilar Shrinking Curves for Anisotropic Curvature Flow Equations(Variational Problems and Related Topics). 数理解析研究所講究録 1995, 911: 75-83

ISSUE DATE:

1995-05

URL:

http://hdl.handle.net/2433/59551

RIGHT: 


\section{Existence of Selfsimilar Shrinking Curves for Anisotropic Curvature Flow Equations}

Noriko Mizoguchi

\author{
溝口紀子（東京学芸大·教育）
}

\section{Introduction}

This is a joint work with Prof. C. Dohmen and Prof. Y. Giga.

We consider a simple looking ordinary differential equation of the form

$$
u_{x x}+u-\frac{a(x)}{u}=0 \quad \text { in } \mathbf{R}
$$

with a given positive function $a$. This equation arises in describing a selfsimilar solution of anisotropic curvature flow equations. Since $x$ is the argument of the normal of the curve it is natural to impose $2 \pi$-periodicity for $a$ in (1) and to ask for existence of $2 \pi$ periodic solutions. To simplicity the notation we notice that a $2 \pi$-periodic function can be regarded as a function on the flat torus $\mathbf{T}=\mathbf{R} / 2 \pi \mathbf{Z}$. For example the space $C^{m}(\mathbf{T})$ is the space of all $2 \pi$-periodic $C^{m}$-functions on $\mathbf{R}$. Let $C_{+}^{m}(\mathbf{T})$ denote the set of all positive functions in $C^{m}(\mathbf{T})$. In particular

$$
C_{+}^{2}(\mathbf{T})=\left\{u \in C^{2}(\mathbf{R}): u(x+2 \pi)=u(x) \text { for } x \in \mathbf{R}, u>0\right\}
$$

Using this notations, we want to investigate the existence of solutions of $(1)$ in $C_{+}^{2}(\mathbf{T})$. As to this, we have the following

Theorem 1. Assume that $a$ is a positive, continuous function on $\mathbf{T}$. Then there is a function $u \in C_{+}^{2}(\mathbf{T})$ solving $(1)$. 
The key step to prove this result is to derive a priori bounds for solutions of (1) :

Theorem 2. Let $0<A_{1}<A_{2}$ be two constants. Then there are two positive constants $m$ and $M$, depending only on $A_{1}$ and $A_{2}$, such that if $u \in C_{+}^{2}(\mathbf{T})$ solves (1) on $\mathbf{T}$ with

$$
A_{1} \leq a \leq A_{2}
$$

then

$$
m \leq u \leq M \quad \text { on } \mathbf{T}
$$

The proof of this a priori estimate actually shows that the continuity of $a$ is not needed.

Corollary 1. Let $a \in L^{\infty}(\mathbf{T})$ and satisfy (3). Then there is a function $u \in C_{+}^{1,1}(\mathbf{T})$ solving (1).

Here $C_{+}^{1,1}(\mathbf{T})$ denotes the space of all positive, $2 \pi$-periodic functions whose derivative is Lipschitz continuous. The differential equation is solved in the sense of distributions and almost everywhere.

To prove this corollary, we approximate $a$ by continuous functions $a_{j}$, keeping the bounds (3) and $a_{j} \rightarrow a$ in $L_{l o c}^{p}$-sense for $p>1$ as $j \rightarrow \infty$. Let $u_{j}$ be the solution of (1) taking $a_{j}$ instead of $a$. By the a priori bounds (4) and the equation (1) the sequence $u_{j}$ is bounded in $L^{\infty}$ along with $u_{j x}$ and $u_{j x x}$. Thus a subsequence of the $u_{j}$ converges to some function $u$ in $C_{+}^{1}(\mathbf{T})$; it is not difficult to show $u \in C_{+}^{1,1}(\mathbf{T})$ and that $u$ solves (1).

To get a better understanding of the mechanisms we will carry out the proof of the a priori bounds considering the slightly more general equation

$$
u_{x x}+u-a(x) g(u)=0 \quad \text { in } I \subset \mathbf{R}
$$

instead of (1). Here again a satisfies (3) on the interval $I$ and $g$ is assumed to be a positive, continuous, nonincreasing function on $(0, \infty)$. Defining

$$
G(p)=\int_{1}^{p} g(s) d s
$$


we consider impose the following conditions on $g$ :

$$
\begin{gathered}
\lim _{p \rightarrow 0} G(p)=-\infty, \quad \lim _{p \rightarrow \infty} G(p) p^{-2}=0, \\
\lim _{p \rightarrow 0, q \rightarrow \infty} \frac{G(p) p}{g(q) q^{2}}=0 \\
\lim _{p \rightarrow \infty} g(p)=0 .
\end{gathered}
$$

Note that the second condition in (7) is automatically satisfied by (6) and the nonincreasing property of $g$. Examples for functions satisfying these conditions are given by

$$
g(p)=p^{-\sigma}, \quad 1 \leq \sigma<2
$$

Our main existence theorem has an application for evolution equations for embedded colsed curves $\left\{\Gamma_{t}\right\}_{t>0}$ in $\mathbf{R}^{2}$ derived in [10].

Let $V$ be the inward velocity of $\Gamma_{t}$ in the direction of its unit inward normal vector

$$
n(\theta)=(\cos \theta, \sin \theta)
$$

Let $k$ be the inward curvature of $\Gamma_{t}$ and let $f$ and $\beta$ be positive functions on $\mathbf{R}$, which are $2 \pi$-periodic. we consider an equation for $\Gamma_{t}$ of the form

$$
V=a(\theta) k, \quad a(\theta)=\frac{f^{\prime \prime}(\theta)+f(\theta)}{\beta(\theta)} .
$$

Here $f^{\prime \prime}+f$ is assumed to be positive so that the equation is parabolic. Such an equation arises in a model describing the motion of phase boundaries in an anisotropic medium (see $[10]$ ). The function $f$ is called the surface energy density and $\beta$ is called the cinetic coefficient.

If $a(\theta)$ is constant, the equation becomes the curvature flow equation and the evolution of $\Gamma_{t}$ is well studied. No matter what initial curve is given, the solution stays smooth and embedded and eventually becomes convex ([10]). It then stays convex and 
shrinks to a point in finite time ([8]). The type of shrinking is asymptotically similar to that of a shrinking circle $\left\{C_{t}\right\}([6],[7],[8])$, which is self-similar in the sense that

$$
C_{t}=\left(t_{*}-t\right)^{1 / 2} C
$$

where $C$ denotes the unit circle centered at the origin, the time $t_{*}$ is the extinction time and $\lambda C$ denotes the dilatation of $C$ with multiplier $\lambda$. Selfsimilar solutions are classified even for immersed curves ([2]) and the asymptotic shape of singularities of this type is classified ([1]). We are interested in finding such selfsimilar solutions

$$
\Gamma_{t}=\left(t_{*}-t\right)^{1 / 2} \Gamma
$$

for general $a(\theta)$. Such solutions exist in the case that $\beta(\theta)^{-1}$ equals a constant multiple of $f(\theta)$. Then $\Gamma$ is the boundary of the so-called Wulff-shape $W$ of $f$, i.e.,

$$
W=\left\{x \in \mathbf{R}^{2}: x \cdot n(\theta) \leq f(\theta) \text { for all } \theta \dot{\epsilon} \mathbf{R}\right\}
$$

This is explicitely stated in [12], including the multidimensional case where $\beta$ and the second differential $f^{\prime \prime}$ are assumed continuous, so also $a$ is continuous. It is not difficult to see that such results extend to $f \in C^{1,1}$, provided that $f^{\prime \prime}+f$ is still bounded away from zero and if the definition of a solution is given in some appropriate sense.

Our main existence theorem yields the existence of selfsimilar solutions for arbitrary bounded $a$. Indeed every equation $V=a(\theta) k$ can be rewritten as

$$
V=u\left(u^{\prime \prime}+u\right) k
$$

where $u$ is a solution of (1) with $\theta$ replacing $x$.

\section{A priori extimates}

To simplify the terminology let us difine the following terms. A solution $u \in C_{+}^{2}(\mathbf{T})$ of (1) or (5) is called a singlepeak-solution if the set of points not being local extrema consists of two connedted components in T. Otherwise $u$ is called a multipeak-solution. 
To prove the a priori bounds these two types of solutions need essentially different techniques. Thus let us state the results separately.

Lemma 1. Let $u \in C_{+}^{2}(I)$ be a solutions of (5) on some open interval $I$ and let (3) be satisfied. If $u$ attains local minima in $\alpha, \beta \in I, \alpha<\beta$ and $n_{x}$ changes its sign only once in $(\alpha, \beta)$, then there is a positive constant $M_{0}$ depending only on $A_{1}, A_{2}$ and $g$ such that

$$
u \leq M_{0} \quad \text { in }(\alpha, \beta)
$$

provided that $\beta-\alpha \leq \pi$.

Lemma 2. Let $u \in C_{+}^{2}(\mathbf{T})$ be a singlepeak-solutions of (5) and let (3) be satisfied. Then there is a positive constant $M_{1}$ depending only on $A_{1}, A_{2}$ and $g$ such that

$$
u \leq M_{1} \quad \text { in } \mathbf{T}
$$

Proposition 1. Let $u \in C_{+}^{2}(\mathbf{T})$ be a solution of (5) and let (3) be satisfied.

i) If there is a constant $\dot{M}$ depending only on $A_{1}, A_{2}$ and $g$ such that one local maximum $u(\gamma)$ is estimated by $u(\gamma) \leq \tilde{M}$, then there are two other constants $0<m<M$, also depending only on $A_{1}, A_{2}$ and $g$ such that

$$
m \leq u \leq M \quad \text { on } \mathbf{T}
$$

ii) The conclusion in i) also holds if there is a constant $\tilde{m}>0$ depending only on $A_{1}, A_{2}$ and $g$ such that one local minimum $u(\alpha)$ is estimated by $u(\alpha) \geq \tilde{m}$.

See the proofs of Lemmas 1, 2 and Proposition 1.in [4]. Theorem 2 is an immediate consequence of Lemma 1, 2 and Proposition 1 as can be seen as follows. If $u$ is a multipeak solution, there exists at least one pair of local minima with a distance less or equal $\pi$. On these intervals Lemma 1 can be applied and due to Proposition 1 all extrema are estimated in terms of one extremum. The situation needed to apply Lemma 
1 fails to exist only if $u$ has exactly one local minimum, i.e., is a singlepeak solution. But in this case Lemma 2 yields the upper bound and due to Proposition 1 we again have a lower bound; thus the theorem is proved.

The results above also show that the set of all $2 \pi$-periodic solutios of (1) or (5) is bounded uniformly in the set of all $a$ that satiafy (3).

\section{Existence of solutions}

In this chapter, we will prove the existence of a solution of (1) using the Leray-Schauder degree. Herein we make use of the uniform boundedness of solutions of (1) with respect to functions $a$ satisfying (3) stated in Theorem 2. We define

$$
E=\left\{v \in C_{+}^{0}(\mathbf{T}): \frac{m}{2} \leq v \leq 2 M \quad \text { in } \mathbf{T}\right\}
$$

Let $F$ be a continuous mapping from $E \times[0,1]$ into $C_{+}^{0}(\mathbf{T})$ defined by

$$
F(u, \tau)=2 u-\frac{\tau a(x)+(1-\tau) a_{0}}{u}
$$

with a constant $a_{0}$ satisfying the bounds imposed on $a$ in (3).

Let $T$ denote a linear compact operator from $C_{+}^{0}(\mathbf{T})$ into itself given by $w=T(f)$, where $w$ is the unique solution of

$$
-w_{x x}+w=f \quad \text { in } \mathbf{T} \text {. }
$$

Setting $S_{\tau}=S(\cdot, \tau)=T \circ F(\cdot, \tau)$, we have a continuous, compact mapping from $E$ into $C_{+}^{0}(\mathbf{T})$. Clearly $u$ is a fixed point of $S_{\tau}$ if and only if $u \in E$ solves

$$
u_{x x}-u+2 u-\frac{\tau a(x)+(1-\tau) a_{0}}{u}=0 \quad \text { in } \mathbf{T}
$$

which is (1) in case of $\tau=1$. The a priori bounds in Theorem 2 now imply that $S_{\tau}$ has no fixed point on the boundary of $E$, in other words

$$
\left(I-S_{\tau}\right) u \neq 0 \quad \text { on } \partial E, \quad 0 \leq \tau \leq 1
$$


Thus the homotopy invarianve of the Leray-Schauder degree yields

\section{Proposition 2.}

$$
\operatorname{deg}\left(I-S_{1}, E, 0\right)=\operatorname{deg}\left(I-S_{0}, E, 0\right) .
$$

To show the existence of a solution of (5) it now suffices to prove that this degree is not equal zero.

Lemma 3. The number

$$
\operatorname{deg}\left(I-S_{0}, E, 0\right)
$$

is not zero ; in fact, it equals -1 .

Proof. As proved by Gage and Hamilton in [8] (see also [2], [5]), there is a unique solution $u \in E$ of

$$
u_{x x}+u-\frac{a_{0}}{u}=0 \quad \text { in } \mathbf{T}
$$

which is given by the constant $a_{0}^{1 / 2}$. (Actually in [8] the setting is $a_{0}=1 / 2$, but our problem here reduces to theirs by changing from $u$ to $\left(2 a_{0}\right)^{1 / 2} u$. )

So $u_{0}=a_{0}^{1 / 2}$ is the only zero of $I-S_{0}$ in $E$; thus

$$
\operatorname{deg}\left(I-S_{0}, E, 0\right)=\operatorname{deg}\left(I-S_{0}, B_{\delta}\left(u_{0}\right), 0\right)
$$

for some sufficiently small $\delta$. At $u_{0}$ the mapping $I-S_{0}$ is nondegenerate in the sense that the derivative $I-S_{0}^{\prime}\left(u_{0}\right)$ is injective. Indeed, suppose that

$$
\left(I-S_{0}^{\prime}\left(u_{0}\right)\right) v=0 .
$$

Since $S_{0}^{\prime}\left(u_{0}\right)=T \circ F^{\prime}\left(u_{0}, 0\right)$, this implies

$$
-v_{x x}+v=2 v+\frac{a_{0}}{u_{0}^{2}} v
$$

or, using the definition of $u_{0}$

$$
v_{x x}+2 v=0 .
$$


But this problem has no nontrivial $2 \pi$-peiodic solution. This nondegeneracy enables us to apply a standard degree theory result (see [11], Theorem 2.8.1, p.66 or [3], Example 2.8 .3$, p.65), which states

$$
\operatorname{deg}\left(I-S_{0}, B_{\delta}\left(u_{0}\right), 0\right)=(-1)^{\beta}
$$

where $\beta$ is the number of eigenvalue of $S_{0}^{\prime}$ (counting algebraic multiplicity) greater than one.

We show the elementary computation of $\beta$. A number $\lambda$ is an eigenvalue of $S_{0}^{\prime}\left(u_{0}\right)$ if and only if there is a nontrivial solution $v \in C_{+}^{0}(\mathbf{T})$ of

$$
\lambda v=S_{0}^{\prime}\left(u_{0}\right) v
$$

or equivalently

$$
-v_{x x}=\frac{3-\lambda}{\lambda} v
$$

Thus $\beta$ equals the number of $\lambda>1$ (counted with multiplicity) that solve $\frac{3-\lambda}{\lambda}=n^{2}$ for some integer $n \geq 0$. As these $\lambda$ are given by $\lambda=3$ and $\lambda=3 / 2$ with multiplicity 1 and 2 , respectively, we have

$$
\operatorname{deg}\left(I-S_{0}, B_{\delta}\left(u_{0}\right), 0\right)=(-1)^{3}=-1 .
$$

Remark 1. Concerning the uniqueness of solutions of (1) in $C_{+}^{2}(\mathbf{T})$, the implicit function theorem implies that the zero of $I-S_{\tau}$ is unique provided $\tau$ is small since no bifurcation from $\left(u_{0}, 0\right)$ occurs due to the nondegeneracy of the unique zero $u_{0}$ of $I-S_{0}$.

\section{References}

[1] S. Angenent, On the formation of singularities in the cruve shortening flow, J. Diff. Geometry 33 (1991), 601-633. 
[2] U. Abresch and J. Langer, The normalized curve shortening flow and homothetic solutions, J. Diff. Geometry 23 (1986), 175-196.

[3] K. Deinling, Nonlinear Functional Analysis, Springer, Heidelberg, Berlin, New York (1985).

[4] C. Dohmen, Y. Giga and N. Mizoguchi, Existence of selfsimilar shrinking curves for anisotropic curvature flow equations, preprint.

[5] C. Epstein and M. Weinstein, A stable manifold theorem for the curve shortening equation, Comm. Pure Appl. Math. 40 (1987), 119-139.

[6] M. Gage, An isoperimetric inequality with application to curve shortening, Duke M. J. 50 (1983), 1225-1229.

[7] M. Gage, Curve shortening makes convex curves circular, Inv. Math. 76 (1984), 357-364.

[8] M. Gage and R. S. Hamilton, The heat equation shrinking convex plane curves, J. Diff. Geometry 23 (1986), 69-96.

[9] The heat equation shrinks embedded plane curves to points, J. Diff. Geometry 26 (1987), 285-314.

[10] M. E. Gurtin, Thermodynamics of evolving phase boundaries in the plane, Clarendon Press, Oxford (1993).

[11] L. Nirenberg, Topics in nonlinear functional analysis, Lecture Notes 1973/74, Courant Inst. of Math. Sciences.

[12] H. M. Soner, Motion of a set by the curvature of its boundary, J. Diff. Eq. 101 (1993), 313-392. 